

\title{
Conjectures on the Size of Constellations Constructed from Direct Sums of PSK Kernels
}

\author{
Matthew G. Parker ${ }^{\star \star}$ \\ Department of Informatics, University of Bergen, N-5020 Bergen, Norway, \\ matthew@ii.uib.no
}

\begin{abstract}
A general equation is given for the size of complex constellations constructed from the direct sum of PSK-like constellation primitives. The equation uses a generating function whose numerator is a power of a 'coordination polynomial'. Conjectures are also given as to the form and value of these coordination polynomials for various PSK. The study has relevance to error-coding, polynomial residue number theory, and the analysis of random walks.
\end{abstract}

\section{Introduction}

Communications systems often transmit data by modulating using Binary or Quaternary Phase Shift Keyed (BPSK or QPSK) or Quadrature Amplitude Modulated (QAM) constellations in the complex plane. But larger constellations can be more bandwidth-efficient and lead to efficient hardware implementation of complex arithmetic and algorithms [1,2]. This paper considers the problem of finding the size of constellations constructed from direct sums of \{PSK plus the origin $\}$, referred to here as 'PSK $\oplus$ ' constellations. These constellations form lattices for $1,2,3$, or 6 PSK primitives, but for any other PSK $\oplus$ there will be residue 'folding' making the determination of constellation size more complicated. This problem can be recast, for $m \mathrm{PSK} \oplus$, as finding an expression for the number of non-identical polynomial residues resulting from the reduction, mod $\Phi_{m}(x)$, of polynomials in $x$ of Coefficient Weight $\leq n$, (for some positive integer, $n)$, and degree $<m$, where $\Phi_{m}(x)$ is the $m^{\text {th }}$ cyclotomic polynomial in $x$. Although residue folding is, for many applications, undesirable, it is hoped that an algebraic understanding of PSK $\oplus$ will help in the construction of constellations more suited to communications systems which use $\operatorname{PSK} \oplus$ as building blocks. Also, from an algebraic point of view, it is useful to be able to enumerate the residues of polynomials, $\bmod \Phi_{m}(x)$. The theorem and conjectures to be presented here are based on computational results. During the course of the work integer sequences, relating to the $8 \mathrm{PSK} \oplus$ and $16 \mathrm{PSK} \oplus$ constellations, were entered into Sloane's On-Line Encyclopedia of Integer Sequences [3] and were found to refer, in particular, to the paper by Conway and Sloane on Low Dimensional Lattices [4] which, in turn, references work by O'Keefe [5] and others [6].

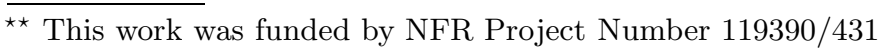


Their results have applications to crystallography, and use generating functions which require the specification of a 'Coordination Sequence'. This paper conjectures a general solution to a related problem, although a general form for the Coordination Sequence (Polynomial) has yet to be found. The results could be used to help extend the scope of error coding strategies such as $[7,8]$, and may also be useful for the development of 'Random Walk' statistics.

\section{Statement of the Problem}

Define $m \mathrm{PSK}+$ as the set of $m+1$ points in the complex plane given by,

$$
m \mathrm{PSK}+=\left\{0,1, w, w^{2}, \ldots, w^{m-1}\right\}
$$

where $w=e^{\frac{2 \pi i}{m}}$, and $i^{2}=-1$. Define $m \operatorname{PSK} \oplus n$ as the direct sum of $n$ copies of $m \mathrm{PSK}+$, given by,

$$
m \mathrm{PSK} \oplus n=\sum_{k=0}^{n-1}\left\{0,1, w, w^{2}, \ldots, w^{m-1}\right\}
$$

We wish to find a formula for $d_{n}$ as $n$ varies over the positive integers, where $d_{n}$ is the number of non-identical points in $m \mathrm{PSK} \oplus n$, given by,

$$
d_{n}=\left|\sum_{k=0}^{n-1}\left\{0,1, w, w^{2}, \ldots, w^{m-1}\right\}\right|
$$

For instance, let $m=4$. The kernel constellation is $\left\{0,1, w, w^{2}, w^{3}\right\}$, where $w=e^{\frac{2 \pi i}{4}}$, and,

$$
d_{2}=\left|\sum_{k=0}^{1}\left\{0,1, w, w^{2}, w^{3}\right\}\right|=|\{0, \pm 1, \pm w, \pm 1 \pm w, \pm 1 \mp w, \pm 2, \pm 2 w\}|=13
$$

As another example, for $m=6$ and $n=2$,

$$
d_{2}=\left|\left\{0, \pm 1, \pm w, \pm w^{2}, \pm 2, \pm 2 w, \pm 2 w^{2}, \pm 1 \pm w, \pm 1 \mp w^{2}, \pm w \mp w^{2}\right\}\right|=19
$$

An algebraic description of the same problem is as follows.

Definition 1 The 'Coefficient Weight', (cw), of a polynomial, $f(x)$, is the sum of it's coefficient values. In other words $c w(f(x))=f(1)$.

Let $g(x)=\sum_{i} g_{i} x^{i}$. Let,

$$
\mathbf{G}_{\mathbf{m}, \mathbf{n}}=\left\{g(x) \mid 0 \leq \operatorname{deg}(g(x))<m, g_{i} \geq 0 \quad \forall i, 0 \leq \operatorname{cw}(g(x)) \leq n\right\}
$$

where $\operatorname{deg}(a(x))$ is the degree of $a(x)$. Let $x=e^{\frac{2 \pi i}{m}}$, where $i^{2}=-1$. Then,

$$
m \mathrm{PSK} \oplus n=\left\{h(x) \mid h(x)=\langle g(x)\rangle_{\Phi_{m}(x)}, \forall g(x) \in \mathbf{G}_{\mathbf{m}, \mathbf{n}}\right\}
$$

where $\langle a\rangle_{b}$ is the residue of $a \bmod b$, and $\Phi_{m}(x)$ is the $m^{\text {th }}$ cyclotomic polynomial. Therefore,

$$
d_{n}=|m \operatorname{PSK} \oplus n|
$$

as before. 


\section{Computational Results}

Tables 1 and 2 show some computed values of $d_{n}$ for various $n$ and $m$. The number of Euclidean distances, $D$, refers to the size of the set of values for the absolute (straight-line) distance from each point in $m \mathrm{PSK} \oplus n$ to the origin. The figures for $D$ are not discussed further in this paper, but are included here for the reader's interest.

Table 1. Constellation and Euclidean Distance Enumerations for Various $m \mathrm{PSK} \oplus n$ $d_{n}$-No of points in constellation. $D$-No of Euclidean distances.

\begin{tabular}{|c|c|c|c|c|c|c|c|c|c|c|c|c|c|c|c|c|c|c|}
\hline $\begin{array}{l}n \\
m \\
m\end{array}$ & 1 & & 2 & & 3 & & 4 & & 5 & & 6 & & 7 & & 8 & & 9 & 10 \\
\hline 3 & $\frac{a_{n}}{4}$ & $\frac{\Delta}{2}$ & $\frac{x_{n}}{10}$ & $\frac{D}{3}$ & $\frac{a_{n}}{19}$ & $\frac{D}{5}$ & $\frac{d_{n}}{31}$ & $\frac{D}{7}$ & $\frac{d_{n}}{16}$ & $\frac{D}{9}$ & $d_{n}$ & $\frac{D}{D}$ & $\frac{d_{n}}{85}$ & $D$ & $d_{n}$ & \begin{tabular}{l|l}
$D$ \\
\end{tabular} & $\frac{d_{n}}{13}$ & $\frac{d_{n}}{166}$ \\
\hline 4 & $\begin{array}{l}4 \\
5\end{array}$ & 2 & 13 & 4 & 25 & 6 & $\begin{array}{l}31 \\
41\end{array}$ & 9 & $\begin{array}{l}46 \\
61\end{array}$ & 9 & $\begin{array}{l}64 \\
85\end{array}$ & $\begin{array}{l}12 \\
16\end{array}$ & $\begin{array}{l}85 \\
113\end{array}$ & $\begin{array}{l}15 \\
19\end{array}$ & $\begin{array}{l}101 \\
149\end{array}$ & 18 & & 166 \\
\hline 5 & 6 & 2 & 21 & 5 & 56 & 8 & 126 & 17 & & & & & & & & & & \\
\hline 6 & 7 & 2 & 19 & 4 & 37 & 6 & 61 & 9 & 91 & 12 & 127 & 16 & 169 & 20 & & & & \\
\hline 7 & 8 & 2 & 36 & 6 & 120 & 14 & 330 & 30 & & & & & & & & & & \\
\hline 8 & 9 & 2 & 41 & 6 & 129 & 13 & 321 & 29 & 681 & 53 & 1289 & 96 & 2241 & & 3649 & & 641 & 8361 \\
\hline 9 & 10 & 2 & 55 & 6 & 217 & 17 & 685 & 46 & 1837 & 99 & & & & & & & & \\
\hline 10 & 11 & 2 & 61 & 7 & 211 & 77 & 551 & 38 & 1201 & 72 & & & & & & & & \\
\hline 12 & 13 & 2 & 73 & 7 & 253 & 16 & 661 & 38 & 1441 & 72 & & & & & & & & \\
\hline 14 & 15 & 11 & 13 & 9 & 575 & 29 & 2171 & 96 & & & & & & & & & & \\
\hline 15 & 16 & 13 & 36 & 9 & 811 & 33 & 3751 & 132 & 14176 & 440 & & & & & & & & \\
\hline 16 & 17 & 14 & 45 & 10 & 833 & 35 & & & & & & & & & & & & \\
\hline 18 & 19 & 16 & 63 & 10 & 865 & 33 & 3313 & 114 & & & & & & & & & & \\
\hline 20 & 21 & 22 & 21 & 12 & 1521 & 46 & & & & & & & & & & & & \\
\hline 21 & 22 & 25 & 53 & 12 & 2017 & 59 & 12496 & 322 & 63946 & 1396 & & & & & & & & \\
\hline 22 & 23 & 26 & 65 & 13 & 2047 & 59 & 11969 & 310 & & & & & & & & & & \\
\hline 24 & 25 & 28 & 899 & 13 & 2089 & 54 & 10825 & 258 & & & & & & & & & & \\
\hline 25 & 26 & 35 & 351 & 15 & 3276 & 78 & & & & & & & & & & & & \\
\hline 27 & 28 & 40 & 106 & 15 & 4051 & 89 & 31213 & 4296 & & & & & & & & & & \\
\hline 30 & 31 & $4 !$ & 51 & 16 & 3901 & 81 & 22831 & 425 & & & & & & & & & & \\
\hline 33 & 34 & $5 !$ & 95 & 18 & 7129 & 125 & 65671 & 1072 & & & & & & & & & & \\
\hline 35 & 36 & 66 & 66 & 20 & 8436 & 138 & & & & & & & & & & & & \\
\hline 36 & 37 & 64 & 49 & 19 & 7237 & 118 & & & & & & & & & & & & \\
\hline 40 & 41 & 84 & 44 & 22 & 11441 & 161 & & & & & & & & & & & & \\
\hline 45 & 46 & 10 & 0812 & 24 & 17281 & 213 & & & & & & & & & & & & \\
\hline 48 & 49 & 11 & 1532 & 25 & & & & & & & & & & & & & & \\
\hline 49 & 50 & 12 & 275 & 27 & & & & & & & & & & & & & & \\
\hline 50 & 51 & 13 & 3012 & 27 & 22051 & 246 & & & & & & & & & & & & \\
\hline 54 & 55 & 14 & 459 & 28 & 24949 & 258 & & & & & & & & & & & & \\
\hline 60 & 61 & 18 & 8013 & 31 & 33901 & & & & & & & & & & & & & \\
\hline 75 & 76 & 29 & 926 & 39 & & & & & & & & & & & & & & \\
\hline 90 & 91 & \begin{tabular}{l|l}
2 & 40
\end{tabular} & 051 & 46 & & & & & & & & & & & & & & \\
\hline
\end{tabular}

And here are a few more partial results for the case $m=8$.

Table 2. Constellation Enumerations for More $8 \mathrm{PSK} \oplus n$

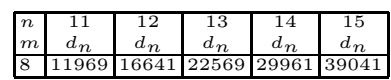

\section{Some Conjectures}

We shall form a generating function for the sequences, $d_{n}$, where $d_{n}$ is different for every $m$. Thus define $d_{m}(x)=\sum_{n=0}^{\infty} d_{n} x^{n}$. The following conjecture satisfies all numerical results quoted above, 


\section{Conjecture 1}

$$
d_{m}(x)=\frac{c_{h}(x)^{\frac{m}{h}}}{(1-x)^{\phi(m)+1}}
$$

where $\phi$ is Euler's Totient Function, $h$ is the square free part of $m$, and $c_{h}(x)$ is referred to as the $h^{\text {th }}$ coordination polynomial. $c_{h}(x)$ is palindromic and $\operatorname{deg}\left(c_{h}(x)\right)=\phi(h)$.

The above conjecture omits to specify exactly the form of $c_{h}(x)$. This is an area of further research. However the following theorem determines $c_{h}(x)$ where $h$ is a prime, and two following conjectures satisfy the computational results for $h=2 p, p$ an odd prime, and $h=15$, respectively,

\section{Theorem 1}

$$
c_{p}(x)=\Phi_{p}(x), \quad \text { p prime }
$$

Theorem 1 was conjectured by the author based on numerical computation. A proof was found by T.Kløve and it is given in Appendix A.

\section{Conjecture 2}

$$
c_{2 p}(x)=\sum_{k=0}^{\frac{p-3}{2}} x^{k}+x^{p-1-k} \sum_{i=0}^{k}\left(\begin{array}{c}
p \\
i
\end{array}\right)+x^{\frac{p-1}{2}} \sum_{i=0}^{\frac{p-1}{2}}\left(\begin{array}{c}
p \\
i
\end{array}\right), \quad p \text { an odd prime }
$$

\section{Conjecture 3}

$$
c_{15}(x)=\left(1+x^{8}\right)+7\left(x+x^{7}\right)+28\left(x^{2}+x^{6}\right)+79\left(x^{3}+x^{5}\right)+130 x^{4}
$$

The following observation was also made,

\section{Conjecture 4}

$$
m \mid\left(\frac{m^{n+1}-1}{m-1}-d_{n}\right)
$$

From the computational results values of $c_{h}(x)$ have also been partially ascertained for various $h$ as shown in Table 3 .

All preceding coordination polynomials were computed from the $d_{n}$ sequences using the following strategy. For instance, for $m=6$ the $d_{n}$ sequence is computed to be $1,7,19,37,61,91,127,169, \ldots$. Thus $d_{6}(x)=1+7 x+19 x^{2}+37 x^{3}+61 x^{4}+91 x^{5}+$ $127 x^{6}+169 x^{7}+\ldots$ Note that $\phi(6)+1=3$ so, from Conjecture 1 , we multiply $d_{6}(x)$ (truncated to degree 7 ) by $(1-x)^{3}$ to get $c_{6}^{\prime}(x)=e(x)+x^{2}+4 x+1$, where $e(x)$ is some error term due to having truncated $d_{6}(x)$ to degree 7 . In this case $e(x)=-217 x^{8}+380 x^{9}-169 x^{10}$, which is evidently an error term so $c_{6}(x)=x^{2}+4 x+1$. The same strategy can be used to compute $c_{h}(x)$ for all $d_{n}$ sequences in the table, and hence arrive at the preceding Conjectures $2-3$ on the form of $c_{h}(x)$. 
Table 3. Incomplete Coordination Polynomials for Various Composite $h$

\begin{tabular}{|l|l|}
\hline$h$ & $c_{h}(x)$ \\
\hline 21 & $1+9 x+45 x^{2}+158 x^{3}+432 x^{4}+909 x^{5}+\ldots ?$ \\
30 & $1+22 x+208 x^{2}+874 x^{3}+1480 x^{4}+\ldots ?$ \\
33 & $1+13 x+91 x^{2}+444 x^{3}+1677 x^{4}+\ldots ?$ \\
35 & $1+22 x+208 x^{2}+874 x^{3}+1480 x^{4}+\ldots ?$ \\
\hline
\end{tabular}

\section{Triangle Patterns}

An examination of number triangles may give a clue as to how to extend the previous conjectures on coordination polynomials to the more general case. On page 14 of [4] it was observed that the coordination polynomials for the dual lattice, $A_{d}^{*}$, satisfy the following 'coordinator' triangle.

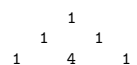

$$
\begin{aligned}
& { }^{1}{ }_{6}^{5}{ }_{16}^{5} \quad 1
\end{aligned}
$$

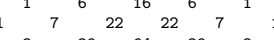

$$
\begin{aligned}
& 1_{9}^{1}{ }^{8}{ }_{37}{ }^{29}{ }_{93}{ }^{64}{ }_{93}{ }^{29}{ }_{37}^{8}{ }^{8} 9^{1} 1 \\
& \begin{array}{llllllllllllllllllll}
1 & 11 & 56 & 176 & 386 & 386 & 176 & 56 & 11 & 1 & 1
\end{array}
\end{aligned}
$$

The $p^{\text {th }}$ line of the above triangle, $p$ prime, also provides the coordination polynomials, $c_{2 p}(x)$, for Conjectures 1 and 2 of this paper.

In the same way we can construct a partial triangle for the $c_{3 p}(x)$ case, using our previous computational results. Thus,

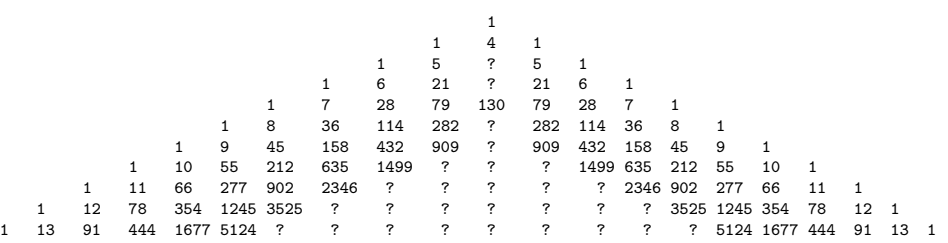

where each entry apart from those of the middle three columns seems to be the sum of the three entries immediately above, e.g. $158=8+36+114$. Note that the only triangle entries directly computed from computational results are the sequences, $1,4,1$, and $1,7,28,79,130,79,28,7,1$, and 1,9,45,158,432,909, and 1,13,91,444,1677. All other numbers in the above triangle are nominally filled in to fit the 'sum of three' conjecture. The $c_{3 p}(x)$ coordination polynomial can be read from the $p^{\text {th }}$ line of the previous triangle for $p$ prime. For instance, $c_{15}(x)=\left(1+x^{8}\right)+7\left(x+x^{7}\right)+$ $28\left(x^{2}+x^{6}\right)+79\left(x^{3}+x^{5}\right)+130 x^{4}$. Although we do not currently have an equation for $c_{3 p}(x)$ it is worth noting that the following triangle is similar to the previous triangle, 


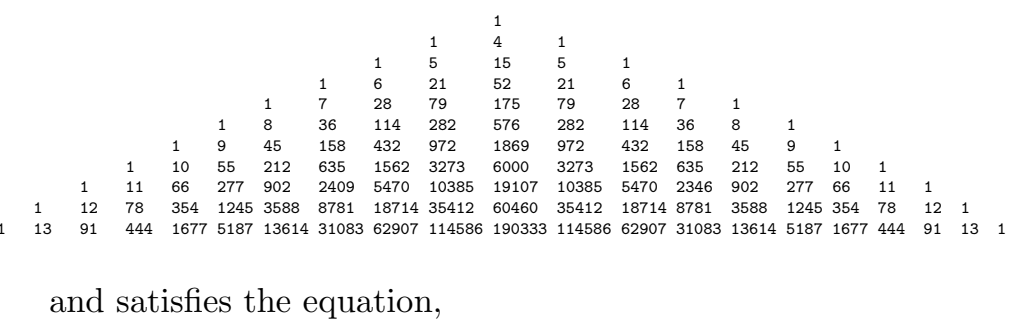

$$
P_{r}(x)=\sum_{k=0}^{r-1}(3 x)^{k}\left(1+x+x^{2}\right)^{r-k-1}
$$

for each line of the triangle, $r$, thus providing a clue as to the true form of $c_{3 p}(x)$.

Appendix B sketches out an alternative strategy for the rapid computation of the coefficients, $d_{n}$, of $d_{m}(x)$ for general $m$, and is a good starting place for further research. It is hoped that the strategy of Appendix B may lead to a proof of the remaining conjectures, in particular Conjecture 1, and may also lead to a theorem for the construction of $c_{h}(x)$ in the general case.

\section{Conclusion}

This paper has presented computational results relating to the size of constellations formed from direct sums of PSK-type constellations. A theorem and a number of conjectures have been offered, comprising formulae for the rapid computation of sizes of such 'direct-sum' constellations. These formulae have application to error-control coding, random-walk statistics, algebraic number representations, and (polynomial) residue number theory. It remains to verify the conjectures.

\section{Appendix A- Proof of Theorem 1 by T.Kløve}

Let $p_{r}(n)$ denote the number of ordered partitions of $n$ into $r$ parts, that is

$$
p_{r}(n)=\left|\left\{\left(a_{1}, a_{2}, \ldots, a_{r}\right) \in N^{r} \mid a_{1}+a_{2}+\ldots+a_{r}=n\right\}\right|
$$

where $N=\{0,1,2, \ldots\}$. If,

$$
n=a_{1}+a_{2}+\ldots+a_{r-1}+a_{r}
$$

and $a_{i} \geq 1$ for $i=1,2, \ldots, r-1$, then

$$
n-(r-1)=\left(a_{1}-1\right)+\left(a_{2}-1\right)+\ldots+\left(a_{r-1}-1\right)+a_{r}
$$

and vice versa. Hence the number of ordered partitions of $n$ into $r$ parts such that the first $r-1$ parts are positive is $p_{r}(n-(r-1))$. 
Lemma 1 We have

$$
\sum_{n=0}^{\infty} p_{r}(n) x^{n}=\frac{1}{(1-x)^{r}} \quad \text { and } \quad \sum_{n=r-1}^{\infty} p_{r}(n-(r-1)) x^{n}=\frac{x^{r-1}}{(1-x)^{r}}
$$

Proof of Lemma 1: These are standard results from the theory of partitions:

$$
\sum_{n=0}^{\infty} p_{r}(n) x^{n}=\left(1+x+x^{2}+x^{3}+\ldots\right)^{r}=\frac{1}{(1-x)^{r}}
$$

and

$$
\sum_{n=r-1}^{\infty} p_{r}(n-(r-1)) x^{n}=x^{r-1} \sum_{n=r-1}^{\infty} p_{r}(n-(r-1)) x^{n-(r-1)}=\frac{x^{r-1}}{(1-x)^{r}} .
$$

Lemma 2 Let $m$ be an odd prime. Then $d_{n}=p_{m+1}(n)-p_{m+1}(n-m)$.

Proof of Lemma 2: $d_{n}$ counts the number of distinct sums

$$
a_{1} w+a_{2} w^{2}+\ldots+a_{m} w^{m}+a_{m+1} \cdot 0
$$

where $a_{i} \geq 0$ for $i=1,2, \ldots, m+1$ and $a_{1}+a_{2}+\ldots+a_{m+1}=n$. Noting that $w+w^{2}+\ldots w^{m}=0$ we get $d_{n}$ by counting all sums $(1)$, this number is $p_{m+1}(n)$, and subtracting the number of sums where $a_{i} \geq 1$ for $i=1,2, \ldots, m$, this number is $p_{m+1}(n-m)$ (as explained above).

Theorem 1 now follows from the two lemmas:

$$
\begin{aligned}
& \sum_{n=0}^{\infty} d_{n} x^{n}=\sum_{n=0}^{\infty} p_{m+1}(n) x^{n}-\sum_{n=0}^{\infty} p_{m+1}(n-m) x^{n}=\frac{1-x^{m}}{(1-x)^{m+1}}=\frac{\Phi_{m}(x)}{(1-x)^{m}} \\
& \text { since } \Phi_{m}(x)=x^{m}+x^{m-1}+\ldots+1 .
\end{aligned}
$$

\section{Appendix B - A General Strategy for Computing the Size of PSK $\oplus$ Constellations}

Here a technique is proposed for the fast computation of the coefficients of $d_{m}(x)$ in the general case. Hopefully this may lead to a general proof of the conjectures of this paper, and a fast way to construct $c_{h}(x)$ in the general case, at least for $m$ up to some large value. The technique will be illustrated by looking at the case where $m=6$. Note that $\Phi_{6}(x)=x^{2}-x+1$. The steps of the technique are the following subsection headings.

\subsection{Find all Forbidden Binary Patterns}

$\Phi_{6}(x)$ implies the following polynomial equivalences:

$$
x^{2}+1=x \quad \text { pattern is } 101000
$$




$$
x^{3}+1=0 \quad \text { pattern is } 100100
$$

These are the two binary patterns (polynomials) which are 'forbidden' for $m=$ 6 . The forbidden polynomials are the set of polynomials which are equivalent, $\bmod \Phi_{m}(x)$, to polynomials of lower hamming weight. Note that, for example, $x^{2}-x+1$ is not included as a 'forbidden' polynomial as it includes the polynomial $x^{2}+1$ as a sub-polynomial. In general, for $m=2 p, p$ prime, there are only two forbidden polynomials, namely, $x^{p-1}+x^{p-3}+x^{p-5}+\ldots+x^{2}+1$, and, $x^{p}+1$. More generally, for large, composite $m$, there may be non-binary forbidden polynomials.

\subsection{Enumerate all Length $m$ Binary Words Which Avoid the Forbidden Patterns}

For $m=6$, and for Hamming Weights (hw) 0-6 we have the following cyclically distinct binary strings which avoid the forbidden patterns or any cyclic shift of the forbidden patterns.

$$
\begin{aligned}
& \frac{\mathrm{hw}=0000000}{\mathrm{hw}=1100000} \\
& \hline \mathrm{hw}=2110000 \\
& \hline \mathrm{hw}=3 \text { none } \\
& \hline \mathrm{hw}=4 \text { none } \\
& \hline \mathrm{hw}=5 \text { none } \\
& \hline \mathrm{hw}=6 \text { none }
\end{aligned}
$$

Each string of non-zero Hamming Weight has cyclic shift order 6. We will refer to the set of length $m$ strings which avoid the forbidden patterns as the 'foundation' polynomials. These 'foundation' polynomials form the set $\mathbf{E}$. For $m=6|\mathbf{E}|=3$. We will define there to be $e_{\mathrm{hw}, m}$ cyclically distinct length $m$ binary words in $\mathbf{E}$, $0 \leq \mathrm{hw} \leq m$. For $m=6, e_{0,6}=1, e_{1,6}=1, e_{2,6}=1, e_{3,6}=0, e_{4,6}=0, e_{5,6}=0$, $e_{6,6}=0$. Note that $e_{0, m}=1 \forall m$.

\subsection{Use Each Member of E as a 'Foundation' for Building All Length $m$ Inequivalent Polynomials of Coefficient Weight $n$, $\bmod \Phi_{m}(x)$}

The '1' positions of the 'foundation' polynomials of $\mathbf{E}$ mark the positions where we are allowed to add 'coefficient weight' to construct our inequivalent polynomials. It therefore follows that the number of inequivalent polynomials, $d_{n}$, satisfies,

$$
d_{n}=1+m \sum_{k=1}^{n} \sum_{\mathrm{hw}=1}^{m}\left(\begin{array}{c}
k-1 \\
k-\mathrm{hw}
\end{array}\right) e_{\mathrm{hw}, m}
$$


For $m=6$,

$$
\begin{aligned}
& d_{0}=1 \\
& d_{1}=1+6=7 \\
& d_{2}=1+6+6(1+1)=19 \\
& d_{3}=1+6+6(1+1)+6(1+2+0)=37 \\
& d_{4}=1+6+6(1+1)+6(1+2+0)+6(1+3+0+0)=61 \\
& d_{5}=1+6+6(1+1)+6(1+2+0)+6(1+3+0+0)+6(1+4+0+0+0)=91 \\
& \ldots \text { etc }
\end{aligned}
$$

These numbers agree with those of Table 1 . The number of $r$-way ordered partitions adding to $n$ is $p_{r}(n)$, and

$$
p_{r}(n)=\left(\begin{array}{c}
n+r-1 \\
n
\end{array}\right)
$$

Therefore we can rewrite (2) in terms of partitions as,

$$
d_{n}=1+m \sum_{k=1}^{n} \sum_{\mathrm{hw}=1}^{m} p_{\mathrm{hw}}(k-\mathrm{hw}) e_{\mathrm{hw}, m}
$$

\subsection{Comments on the Technique}

The technique assumes that all polynomials in $\mathbf{E}$ have cyclic order $m$. It seems likely that this is true in general as $d_{n}$ appears to satisfy $m \mid\left(d_{n}-1\right)$ for all cases computed in Tables 1 and 2. A proof of Conjecture 1, and a proof of the general form of $c_{h}(x)$ may well follow if one can do the following for a given $m$,

1. Derive an efficient method to compute the 'forbidden' polynomials.

2. Derive an efficient method to compute the elements $e_{\mathrm{hw}, m}$ of $\mathbf{E}$ from the forbidden polynomials.

For large $m$ (e.g. perhaps $m=105$ ?) there may be non-binary forbidden polynomials for which the above technique must be modified as follows: Consider, as an example, a 'hypothetical' forbidden polynomial, $F(x)$, of the following form:

$$
F(x)=x^{5}+3 x^{2}+x+2
$$

Then it has an associated binary forbidden polynomial, $f(x)$, where,

$$
f(x)=x^{5}+x^{2}+x+1
$$

We wish to disallow all polynomials built from the foundation $F(x)$ not $f(x)$. Let the cyclic order (over $m$ ) of $F(x)$ and $f(x)$ be $v$. Then we should include $\gamma_{n}$ polynomials in our count for $d_{n}$, where

$$
\gamma_{n}=v\left(\sum_{k=1}^{n} p_{4}(k-4)-\sum_{k=1}^{n-3} p_{4}(k-4)\right)=v \sum_{k=n-2}^{n} p_{4}(k-4)
$$


where the ' 3 ' in the summation limit of the previous equation is the coefficient weight (cw) of $F(x)$ minus the hamming weight of $F(x)$. In general, for a given forbidden polynomial $F(x)$ we include $\gamma_{n}$ in our count for $d_{n}$ where $\gamma_{n}$ satisfies,

$$
\gamma_{n}=v \sum_{k=n+\mathrm{hw}(F(x))-\operatorname{cw}(F(x))+1}^{n} p_{\mathrm{hw}(F(x))}(k-\mathrm{hw}(F(x)))
$$

In the case where the forbidden polynomial is a binary polynomial $\mathrm{hw}(F(x))=$ $\operatorname{cw}(F(x))$ and $\gamma_{n}$ for $F(x)$ is 0 , as expected. Things will be further complicated if the cyclic order of $F(x)$ is lower than that of $f(x)$.

\section{Acknowledgements}

The author thanks S.J.Shepherd and D.A.Gillies for helpful discussions, and D.A.Gillies for writing software which independently confirmed results for the $m=8$ case, and provided extra data for this case.

\section{References}

1. Parker, M.G.: VLSI Algorithms and Architectures for the Implementation of Number-Theoretic Transforms, Residue and Polynomial Residue Number Systems. PhD thesis, School of Eng, University of Huddersfield, March 1995

2. Safer, T.: Polygonal Radix Representations of Complex Numbers. Theoretical Computer Science. 210, (1999) 159-171

3. Sloane, N.J.A.: An On-Line Version of the Encyclopedia of Integer Sequences. http://www.research.att.com/ njas/sequences/index.html, The Electronic Journal of Combinatorics. 1, (1994) 1-5

4. Conway, J.H., Sloane, N.J.A.: Low Dimensional Lattices VII: Coordination Sequences. Proc. Royal Soc. A453 (1997) 2369-2389

5. O'Keeffe, M.: Coordination Sequences for Lattices. Zeit. f. Krist. 210, (1995) 905908

6. Grosse-Kunstleve, R.W., Brunner, G.O.: Algebraic Description of Coordination Sequences and Exact Topological Densities for Zeolites. Acta Crystallographica. Section A. A52, (1996) 879-889

7. Huber, K.: Codes Over Gaussian Integers. IEEE Trans. on Inf. Theory. 40, No 1, Jan. (1994) 207-216

8. Huber, K.: Codes Over Eisenstein-Jacobi Integers. Contemporary Mathematics. 168, (1994) 165-179 